\title{
ANÁLISE FUNCIONAL DO COMPORTAMENTO
}

\author{
THE FUNCTIONAL ANALYSIS OF BEHAVIOR
}

Maria Amélia MATOS*

\begin{abstract}
RESUMO
A principal preocupação do analista comportamental é com o uso de unidades funcionais enquanto realizando uma análise do comportamento. As influências que levaram a essa postura são analisadas: um modelo de ciência das ciências naturais, um modelo evolucionista, um modelo empirista, e o modelo funciona lista causal de Mach. Coerentemente, sua análise do comportamento é basicamente uma análise do valor adaptativo desse comportamento em relação a seu meio ambiente. As implicações dessa opção para uma concepção de causalidade do comportamento são analisadas, especialmente tendo em vista as metáforas das relações constantes e das seqüências encadeadas. As limitações lingüísticas existentes são igualmente consideradas. Os passos básicos para uma análise funcional são descritos e então desmembrados em passos menores. Exemplos e estratégias da realização da análise funcional são apresentados, assim como referências para trabalhos de análise básica e aplicada.
\end{abstract}

Palavras-chave: Análise do Comportamento. Análise Funcional. Behaviorismo Radical. Skinner.

\begin{abstract}
Behavior Analysis main concern is with the usage of functional units of behavior. The influences that led to this posture are analyzed: a natural sciences model, an evolutionist model, an empiricist model, and a Machian functionalist model. Accordingly, a functional analysis of behavior is an analysis of its fitness to environmental conditions. The implications of this option to the causality model adopted by Behavior Analysis are considered, specially in relation to rejected metaphors such as the constant relations and the chained sequences ones as well as its linguistic limitations. The basic steps of a functional analysis are described and then broken into smaller molecular actions. Examples and strategies for performing a functional analysis of behavior are given, as well as bibliographical references for both basic and applied analysis.
\end{abstract}

Key-words: Behavior Analysis. Functional Analysis. Radical behaviorism. Skinner.

(*) Ph. D. pela Columbia University of New York, Professora Assistente do Instituto de Psicologia da USP - SP.

Endereço para correspondência: Rua Engenheiro Bianor, 153 - CEP 05502-010 - São Paulo - SP -E-mail: maamatos@usp.br 
O que distingüe um analista do comportamento de outros estudiosos do comportamento? Diante desta questão Per Holth, em um e-mail recente, propôs que a diferença seria a ênfase que o analista de comportamento dá ao uso de unidades funcionais do comportamento como seu objeto de estudo.

O que é uma unidade funcional do comportamento? Como determiná-la? Como estudá-la?

A resposta a essa pergunta provavelmente envolve tanto uma análise das posturas que levaram a essa opção, como uma análise das técnicas específicas de controle experimental que permitem essa determinação e estudo. Este é o resumo do texto a seguir.

\begin{tabular}{|l|}
\hline O analista do comporta - \\
mento investiga e usa \\
unidades funcionais do \\
comportamento
\end{tabular}

O analista comportamental é antes de mais nada um cientista natural, não um filósofo, não um cientista social e muito menos um estudioso do mental. É também um darwinista e um funcionalista. Parafraseando Jack Michael (1993): Ele tem uma postura determinista na medida em que vê o comportamento humano como um produto inevitável de uma herança genética e de eventos ambientais ocorrendo durante a vida de uma pessoa. Acredita firmemente no uso do método científico em relação a todos os aspectos do comportamento humano. Não estuda apenas o condicionamento operante, não exclui eventos privados e comportamentos encobertos de suas análises. Não insiste que o comportamento possa mudar apenas como resultado de uma exposição direta a contingências, reconhecendo que mudanças podem resultar de instrução e de descrições das contingências. Não tem uma postura anti-fisiologia, anti-genética, nem anti-teórica; porém rejeita explicações internas inferidas.

Como darwinista (referência ao teólogo e historiador natural inglês, Charles Darwin) o ana- lista de comportamento acredita que todas as mudanças comportamentais, operantes ou não, resultam de um processo de seleção pelas conseqüências. Isto supõe que o organismo seja dotado de uma sensibilidade inata ao efeito destas conseqüências.

Para que possamos entender essa teoria de evolução do comportamento pela seleção por conseqüências, imaginemos um nosso antepassado remotíssimo, milhões de gerações atrás, um trilobita, chamemo-la Dodo. Dodo, como qualquer organismo vivo, efetua trocas de energia com o ambiente e se multiplica. Dodo contudo não se movimenta, sua troca de energias somente ocorre quando é atingido por alguma substância que seu organismo pode absorver. Muitas gerações depois, um descendente de Dodo, Lolo, sofre uma mutação e pode se movimentar, aleatoriamente contudo. Esta reação, não obstante, aumenta muito as chances de sobrevivência de Lolo e de seus descendentes, movimentando-se aumenta a probabilidade de Lolo atingir um local onde a concentração de substâncias nutritivas seja grande. Um dos descendentes de Lolo, sofre uma nova mutação, ele é capaz de mudar sua forma: quando atingido por um objeto ele apresenta o reflexo de emitir projeções que podem 'envelopar' esse objeto. Essa reação aumenta enormemente a chance de sobrevivência desse descendente de Lolo, por impedir que o objeto nutriente escape. Um outro descendente, Nono, passa a apresentar movimentos dirigidos, à luz por exemplo, ou à mudanças de temperatura, ou a qualquer outro fator ambiental que possa estar associado a grandes concentrações de substâncias nutritivas, ou a locais onde a presença de predadores é rara, etc. Taxias, como as que Nono apresenta, supõem não só sensibilidade às conseqüências de uma ação, mas também sensibilidade à associação destas conseqüências com determinadas condições antecedentes. De uma maneira muito tosca este seria o quadro geral de uma evolução de formas de reagir de um organismo vivo. Evolução essa dirigida por suas conseqüências sobre a aptidão desse organismo, isto é, 
sobre a adaptabilidade desse organismo ao ambiente em que vive.

Outro exemplo: o reflexo de salivação diante de gotas de limão na boca ou diante de um limão sendo fatiado. A salivação diante de um líquido ácido na boca dilui a concentração deste líquido e diminui seu possível dano sobre as mucosas da boca e, eventualmente, de outros órgãos. Salivar ao chupar limão tem pois um alto valor de sobrevivência. Salivar antes de terem as gotas de limão atingido a boca tem um valor de sobrevivência maior, pois previne esses danos. Salivar ao receber limão na boca representa uma vantagem em relação à condição anterior de nãosalivação; salivar antes de receber o limão na boca, ao vê-lo sendo fatiado, representa uma vantagem maior em relação à reação anterior, de salivar apenas quando o limão atinge a boca. $O$ valor de sobrevivência do salivar perante a gota de limão é grande, porém muito maior é o valor de sobrevivência da sensibilidade às associações entre a gota de limão e seu cheiro, ou sua aparência, ou seu desenho, ou seu nome (reagir a associações entre estí mulos como se fossem equivalentes tem, um alto valor adaptativo). $\mathrm{E}$ maior ainda será esse valor se essas reações puderem ser transmitidas (ou por genes ou por práticas culturais). Evidentemente, para que a seleção seja eficaz, deve haver competição no espaço comportamental entre essas reações.

Como cientista natural, o analista de comportamento é um baconiano (referência ao filósofo e cientista inglês, Francis Bacon). Trabalha por observação, classificação e indução (gradual!). Evita generalizações apressadas e indevidas, evita o dogma, evita explicações metafísicas. É basicamente um empirista (estuda casos concretos) e um experimentalista (submete suas explicações a testes e demonstrações com manipulação de variáveis), só secundariamente, é um "interpretacionista". Privilegia uma abordagem histórica aos eventos. Acha que uma explicação do comportamento humano começa com uma explicação do comportamento animal. O comportamento é governado e modificado por associações adquiridas por experiên- cia, e mantidas por sua utilidade biológica (por sua função de sobrevivência e valor adaptativo).

Como funcionalista o analista de comportamento é um machiano (referência ao físico alemão Ernst Mach). Substitui a noção de 'causa' pela de 'função', e a explicação pela descrição. Para um estudioso da natureza não há nada mais do que o estudo da dependência dos fenômenos, uns em relação aos outros. Os fenômenos sempre ocorrem em variadas relações de interdependência uns em relação aos outros e assim a tarefa do estudioso funcionalista é descrever estas interdependências. Causas e efeitos são simplesmente mudanças, correlacionadas, em duas classes de fenômenos.

De fato a noção de causa e efeito pode ser substituída com vantagem e economia pela noção de função matemática. Reflexo para Skinner (já em 1931) é uma relação observada, é a descrição das relações entre estímulo e resposta. Assim como o comportamento, do qual o reflexo é uma analogia, o reflexo é uma relação entre eventos ambientais e eventos comportamentais. É fútil a discussão se existe comportamento sem ambiente, assim como a discussão se existe ambiente sem comportamento. Nesse sentido se diz que o comportamento é dito controlado quando ele está (isto é, quando nós assim somos capazes de descrevê-lo) funcionalmente relacionado a variáveis ambientais (isto é, a eventos físicos e sociais, dentre os quais estão eventos encobertos e outros comportamentos do próprio organismo). Causa é sinônimo de função, que é sinônimo de controle, que é sinônimo de descrição de relações funcionais.

Voltamos a repetir: o modelo de causalidade próprio ao analista do comportamento é um modelo de seleção pelas conseqüências. Para um funcionalista, comportamentos evoluem (isto é, se modificam) porque têm uma função de utilidade na luta pela sobrevivência do indivíduo; evoluem porque de alguma maneira representam um mecanismo de lidar com ambientes 
complexos. Assim por exemplo, um comportamento estranho jamais é dito 'patológico', pelo analista comportamental; se ele ocorre é porque de alguma maneira ele é funcional, tem um valor de sobrevivência. Fazer uma análise funcional é identificar o valor de sobrevivência de determinado comportamento. Por exemplo: o comportamento de auto-agressão não é considerado como manifestação de um processo psicótico, é sim, um conjunto de respostas que permitem o acesso, pelo indivíduo, a conseqüências importantes para ele: conseqüências sensoriais, conseqüências sociais, conseqüências como bens tangíveis, ou conseqüências como a evitação de eventos desagradáveis.

\section{Fazer uma análise fun- cional é identificar a função, isto é, o valor de sobrevivência de um deter- minado comportamento.}

Essa insistência sobre a pesquisa das variáveis das quais o comportamento é função distingue a prática do analista do comportamento de outras práticas em psicologia, práticas estas que preferem uma descrição estrutural do comportamento (estudo da composição e organização de elementos de uma ação) versus o estudo da função desse comportamento (utilidade biológica dessa ação). É importante apontar que as análises funcionais e estruturais são complementares, não é o caso de excluir uma ou outra, mas, em Psicologia a análise funcional é fundamental.

Como é fácil depreender do que foi exposto acima, a análise funcional do comportamento substitui uma análise do comportamento em termos causais. O próprio conceito de causa, em ciência, mudou ao longo dos tempos. Causa para os gregos e romanos era a vontade dos deuses. No século XVIII, na Inglaterra, com Hume, a noção de causa não mais incorporava a noção de um agente ou força impulsionadora.
Para Newton (representante da Física mecanicista) as forças eram os agentes de mudanças, quer na magnitude quer na direção, do movimento; a força da gravidade 'atraía' a maçã para baixo. A Física moderna dispensa com essas noções referindo-se, ao invés, à propriedades da variável independente e à propriedades da variável dependente, tais como massa e aceleração.

Hume mostrou que a percepção de causação envolvia mais do que a percepção de relações constantes (que é o sentido comum da palavra causa). "Joãozinho atirou a pedra e quebrou a vidraça”, esta frase supõe uma relação causal que contém um agente que produziu a vidraça quebrada. A frase representa uma relação utópica, infalível e constante: sempre que Joãozinho, ou qualquer outra pessoa, atirar uma pedra contra a vidraça, esta vai quebrar. $\mathrm{Na}$ verdade, a relação de fato depende de considerações sobre a magnitude do atirar pedra, a distância entre Joãozinho e a janela, o tamanho da pedra, o tipo de vidraça etc., etc.

Uma outra maneira de considerarmos relações causais é através da metáfora de seqüências encadeadas, onde cada elo é contíguo no tempo e no espaço ao outro elo, e assim sucessivamente até o elo final, o efeito. Esta metáfora supõe uma linearidade e uma unidirecionalidade no processo causal que estão longe de serem verdadeiras. De fato, o encadeamento de elos causais é um disfarce que pretende esconder a existência de uma causa primeira, o elo inicial.

Para o analista de comportamento não há um agente iniciador, nem mesmo o ambiente é iniciador, de vez que o analista comportamental estuda relações (insisto e repito) relações funcionais. O organismo, o indivíduo não é um agente, mas tão somente, como diz Skinner (1989), o palco das interações (evidentemente, Skinner também não aceita que o self seja o agente iniciador; para ele, pessoa ou self devem ser considerados como conceitos relativos ao repertório comportamental de um organismo, e não como agentes iniciadores). A propó- 
sito desta questão Skinner (1972) compara a gestação de um poema com a gestação de uma criança. Quem, ou o quê, é a causa? a mãe? o pai? a relação dos dois? a constituição do corpo da mãe? o mecanismo reprodutivo da espécie? o processo evolutivo que permitiu esse mecanismo reprodutivo? Não há um agente iniciador, uma causa única, mas muitas e diferentes variáveis atuando em conjunto. Na verdade, nem sequer podemos falar em várias causas ou múltipla causação, tudo o que encontramos ao investigar um fenômeno são relações entre eventos ou entre variáveis. Por outro lado, estas relações são válidas apenas dentro de determinadas condições, isto é, as leis científicas não são universais; encontrar o contexto dentro do qual elas são válidas é outra tarefa do cientista.

No modelo da análise do comportamento uma causa é substituída por uma mudança na variável (ou variáveis) independente(s), e um efeito é substituído por uma mudança na variável (ou variáveis) dependente(s), o que se constitui na nossa relação funcional. A diferença entre análise funcional e análise causal é que na primeira não mais se fala do comportamento humano em termos de agência, pois não mais é necessário falar em forças internas ou externas que causam uma ação; ação é uma propriedade do organismo vivo. Do mesmo modo, o reflexo não explica como uma causa/causa uma reação; simplesmente descreve que mudanças ocorrem num e noutro lado da relação funcional.

A dificuldade em abandonarmos estas posturas causais é a dificuldade em abandonarmos nossos hábitos lingüísticos. A sintaxe da linguagem ocidental impõe a necessidade de sujeitos para verbos, especialmente aqueles relativos a comportamento. É até aceito dizermos que a digestão se processa, mas o pensamento não, alguém pensa! Assim considerada, a noção de análise funcional é realmente subversiva, ao ponto de Hineline (1980) propor que ao lidar com o comportamento transformemos nomes em gerúndios ou em expressões de ação. O termo 'inteligência' seria substituído por 'resolver problemas' ou 'agir inteligentemente'; 'motivação', por 'motivado'; 'memória', por 'lembrar' ou 'comportar-se em relação a eventos passados'; 'imaginar' ou 'fantasiar', por 'comportar-se na ausência do evento'; e 'comportamento', por 'comportar-se'. Por exemplo: a frase 'Uma criança, quando necessita de atenção ou ajuda, aprende a captar o olhar de adultos', poderia ser re-escrita de maneira mais aceitável da seguinte forma: 'O contato visual com adultos pode se tornar um evento reforçador para uma criança, e também um evento discriminativo estabelecendo a ocasião na qual essa criança poderá vir a ser reforçada por esses adultos'.

Vejamos quais são os passos para chegarmos a essa transformação:

1) A identificação e a descrição do efeito comportamental (definir 'captar o olhar de adultos' e 'necessitar de atenção ou ajuda' versus definir 'estabelecer contato visual com adultos').

2) A busca de uma relação ordenada entre variáveis ambientais (presença ou não de adultos, ocorrência de situações 'problema', intervenções do adulto, solução ou eliminação da situação 'problema', etc.) e variáveis comportamentais relacionadas (i.e., relevantes) a esse efeito (descrever as condições em que ocorrem os comportamentos mencionados em 1 ; descrever as variações nessas condições e possíveis variações nos comportamentos).

3) A formulação de predições confiáveis baseadas nas descrições dessas relações (inferências ou suposições que serão postas a prova, 'presença de adultos' como estímulos discriminativos, 'intervenção de adultos' como estímulos reforçadores, etc.).

4) A produção controlada desses efeitos predizíveis (demonstração da confiabilidade das inferências e/ou aplicação do conhecimento). 
Cinco passos básicos para a realização de uma análise funcional do comportamento.

1. Definir precisamente o comportamento de interesse.

2. Identificar e descrever o efeito comportamental.

3. Identificar relações ordenadas entre variáveis ambientais e o comportamento de interesse. Identificar relações entre o comportamento de interesse e outros comportamentos existentes.

4. Formular predições sobre os efeitos de manipulações dessas variáveis e desses outros comportamentos sobre 0 comportamento de interesse.

\section{Testar essas predições.}

Uma análise funcional leva em conta aspectos do ambiente e a função que o comportamento tem naquele ambiente. Eu posso dizer que um pássaro está andando, voando ou caçando. Ele está andando se ele se desloca no chão, ele está voando se se desloca no espaço aéreo, e ele está caçando se persegue e pega uma presa. O chão, o espaço aéreo e a presa são as referências ambientais; os efeitos comportamentais são o deslocamento e ou a presa alcançada. No exemplo anterior é possível dizer que entre o comportamento da criança e o dos adultos se estabeleceu uma relação funcional denominada 'reforçamento'. O termo 'reforçamento' descreve uma relação entre uma classe de eventos (comportamentos) que mudam em função de outra classe de eventos (conseqüências). O termo 'reforçamento' não se refere pois à uma teoria, e sim à uma descrição de uma relação funcional. Nesse sentido uma relação funcional não é diretamente observável, o que observamos são as mudanças no fenômeno 'comportamento' e mudanças no fenômeno 'ambiente' ou, no caso, 'compor- tamento dos adultos'. A partir destas observações hipotetizamos uma relação entre esses fenômenos e a testamos. A relação funcional 'reforçamento' (assim como qualquer outra) é uma inferência, resultado de uma análise funcional. Uma análise funcional nada mais é pois do que uma 'explicação' de um evento pela descrição de suas relações com outros eventos. 'Explicamos' as mudanças que ocorrem com o comportamento da criança descrevendo como essas mudanças ocorrem ou não, a depender de certas condições do ambiente, como a presença ou não de adultos, a intervenção ou não destes etc., condições denominadas 'eventos antecedentes' e 'eventos conseqüentes'.

Uma análise funcional completa (observação-suposição-verificação) produz uma definição funcional (a definição de reforçamento, por exemplo, 'aumento na probabilidade de ocorrência de um comportamento que é seguido de certas conseqüências'). Uma definição funcional supõe uma análise funcional, mas não é essa análise.

As vantagens de uma análise funcional são que, além de identificar as variáveis importantes para a ocorrência de um fenômeno e, exatamente por isso, permitir intervenções futuras; ela possibilita o planejamento de condições para a generalização e a manutenção desse fenômeno. Ao identificar em que classe de comportamentos uma determinada resposta se inclui, ou em que classe de estímulos uma determinada mudança ambiental se situa, podemos trabalhar com respostas e/ou estímulos equivalentes. Se a resposta identificada nãofor adequada, podemos substituí-la por uma outra mais aceitável, e que, por pertencer à mesma classe, continuará a produzir os mesmos reforçadores que a resposta anterior. Se uma condição ambiental não estiver mais disponível, podemos recorrer a outra condição ambiental equivalente, na certeza de que esta nova condição continuará a exercer o controle desejado sobre a resposta em questão. Uma análise funcional permite até mesmo planejar esta transferência de funções de estímulos 
(Sidman, 1994). Por outro lado, uma análise estrutural por enfocar aspectos de composição (elementos) e organização (disposição, ordem ou seqüência) e dificilmente permitiria planejar essa substituição ou transferência de funções entre eventos.

Outra vantagem de análises funcionais é que elas podem ser realizadas a longo prazo, isto é, entre eventos que estão separados por um intervalo de tempo entre si. Uma determinada variável ambiental pode não estar presente no momento em que ocorreu uma mudança comportamental e mesmo assim estar relacionada com esta mudança. Análises funcionais, por não estarem fundamentadas em aspectos estruturais, permitem uma explicação histórica, e protegem o analista do comportamento de conceitos mediacionistas como memória, informação, trauma, decodificação, complexos etc.

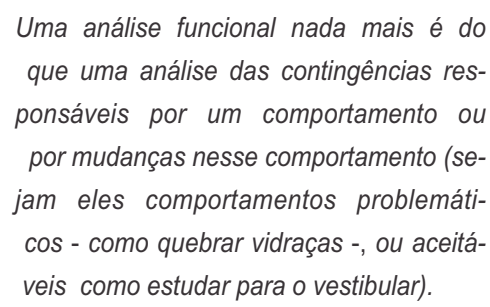

Retomemos nossos cinco passos anteriores, aprofundando sua análise:

1) A identificação do comportamento de interesse envolve sua observação bem como relatos de outras pessoas (pais, professores, outros pesquisadores etc. a depender da situação onde trabalho).

2) A identificação e descrição do efeito comportamental supõem sua especificação, por exemplo, a especificação da freqüência com que ocorre.
3) A busca de uma relação ordenada entre variáveis ambientais e variáveis comportamentais relacionadas (relevantes, instrumentais) com esse efeito supõe:

(a) a descrição da situação antecedente e da situação subseqüente ao comportamento de interesse;

(b) aidentificação da situação subseqüente deve distinguir quais eventos nessa situação são conseqüências;

(c) a identificação da situação antecedente deve distinguir quais eventos nessa situação são de fato condições.

Estas identificações, b e c, podem exigir, e freqüentemente exigem, repetidas observações.

4) A natureza das relações de consequenciação deve ser descrita dentro de um quadro mais amplo (deve ser colocada dentro de um referencial conceitual) pelas vantagens óbvias que isso representa em termos de 'empréstimos' possibilitados: trata-se de reforço positivo ou negativo? de punição? de fuga? de esquiva? A classificação dessas relações em quadros mais amplos permitirá a previsão de ocorrência semelhantes, permitirá o uso de procedimentos de análise já testados, permitirá a comparação com análises anteriores. Do mesmo modo é necessário identificar se as condições antecedentes envolvem apenas eventos físicos ou também comportamentos, e nesse caso se apenas da própria pessoa ou de outras pessoas.

5) E finalmente a análise funcional supõe um teste das previsões. Isso pode representar uma intervenção clínica, 
educacional, institucional ou uma investigação em laboratório.

Os passos 3 e 4 supõem algumas estratégias que, de novo, serão utilizadas para o passo 5. São os chamados 'delineamentos experimentais' (para maiores detalhes ver Sidman, 1960, e Johnston \& Pennypacker, 1980) e todos envolvem o sujeito como seu próprio controle. Como essas estratégias são o 'coração' da Análise Experimental do Comportamento, vale a pena revê-las rapidamente.

a) Delineamento de linha de base simples: dados são coletados sobre o comportamento de interesse antes de qualquer intervenção (fase de linha de base) para uma comparação com os resultados da intervenção (fase de introdução da variável independente). Importantes são a duração da coleta (i.e., da observação) e sua freqüência (i.e., do intervalo entre as observações). Trata-se de um delineamento fraco a ser usado apenas quando prevê-se que a intervenção terá um efeito realmente forte.

b) Delineamento de reversão: as situações de linha de base e intervenção são revertidas (ou alternadas), por esta razão este delineamento é também denominado 'delineamentoA-B-A'. Trata-se de um delineamento de aplicação restrita, ou seja, apenas quando os efeitos da intervenção podem ser cancelados, ou quando o retorno às condições de linha de base representa uma situação socialmente aceitável para o sujeito

c) Delineamento de linha de base múltipla: uma linha de base é estabelecida para cada um de diferentes comportamentos, e uma intervenção é planejada para cada um destes comportamentos, mas esta intervenção é introduzida em diferentes momentos. Importante aqui é decidir sobre qual será duração da observação do efeito de uma intervenção, antes de introduzir uma outra intervenção.

d) Delineamento com mudanças de critério: após a linha de base, uma intervenção é introduzida com um determinado critério de desempenho; após a obtenção/estabilização desse desempenho, o critério é alterado, e assim sucessivamente. Evidentemente que pode ocorrer aqui uma interação entre a natureza da variável que está sendo manipulada na intervenção, e a duração do processo de intervenção, e/ou o número de repetições ou de intervenções, e/ou os critérios de desempenho adotados.

e) Delineamento de tratamentos alternativos: após a linha de base para um determinado comportamento, diferentes intervenções se alternam, segundo uma seqüência determinada, sempre intercaladas com reversões à linha de base (A-B-A-C-A-B-A-D etc.). Neste delineamento há sempre o perigo de interações entre os efeitos das diferentes intervenções.

Resumindo:

Uma análise funcional, sendo uma análise das contingências responsáveis por um comportamento, basicamente busca responder à seguinte questão: "Qual a função deste comportamento para aquela pessoa?", ou, posto de outro modo, "Qual é a relação funcional entre esse comportamento e seus efeitos?"

Evidentemente esta mesma pergunta pode ser feita, mutatis mutantis, quando o comportamento de interesse não ocorre: "Qual é a função da omissão deste comportamento?", ou, de novo, "Qual é a relação funcional entre esse comportamento e seus efeitos?" 


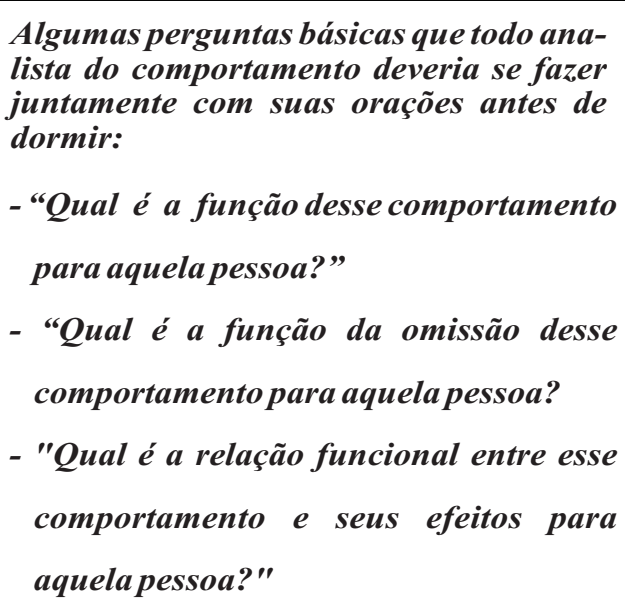

Para terminar, uma palavra de advertência aos que pretendem utilizar a análise funcional na terapia. O problema com a terapia, comportamental ou não, é que este tipo de intervenção lida quase exclusivamente com as especificações verbais do comportamento de interesse (especificações essas que são de fato comportamentos, mas de outro interesse). Isto pode resultar numa psicologia/psicoterapia de poltrona. Verbalizações 'de poltrona' especificam corretamente o ambiente apenas até o ponto em que tatos corretos foram instalados, e apenas até o ponto em que estes tatos permaneçam precisos e corretos. O problema é que operantes verbais de tatos permanecem precisos e corretos somente até o ponto em que metodicamente interajam com as condições (antecedentes e conseqüentes) ambientais 'extra-poltrona' que os instalaram.

Sugerimos a seguir, à guisa de 'receita', alguns passos a serem seguidos quando da realização de uma análise funcional de um determinado comportamento de interesse (Malott, Whaley \& Malott, 1997).

1. Identifeque o comportamento de interesse

a) Verifique se você o enunciou em termos empíricos (Isto é, em termos de ações do participante. Ex.: a criança grita e bate o pé, grita e esperneia, berra e se joga no chão, etc.) e conceituais (Isto é, em termos de classes de ações. Ex.: a criança faz birra). Identifique exemplos e não-exemplos para uma definição completa.

b) Verifique a freqüência de ocorrência de cada uma das ações identificadas, bem como da classe geral.

2. Especifique o produto de cada uma dessas ações e o produto da classe de ações.

a) É uma condição reforçadora ou uma condição aversiva?

b) Sua ação se faz por apresentação, remoção, ou impedimento?

c) O produto é grande, provável, imediato?

d) Existem produtos a longo prazo? Quais?

e) Os produtos são conseqüências naturais ou sociais? São conseqüências mediadas por agentes sociais? Quem são os agentes?

3. Identifique as condições antecedentes necessárias e/ou presentes quando 0 comportamento ocorre.

a) Identifique as condições motivadoras. Identifique as operações estabelecedoras que afetam as condições motivadoras.

b) Especifique os estímulos discriminativos. Existe mais de um discriminativo para um mesmo comportamento? Existe uma classe de estímulos discri-minativos?

c) Os discriminativos são circunstancias e/ou eventos físicos ou sociais?

d) Existem outros compoftamento que ocorrem antes do comportamento de interesse? Sua relação com o comportamento de interesse é de necessidade (pré-requisitos), de facilitação, ou são ocorrências acidentais? 
4. Ordene os comportamentos pré-correntes (necessários e facilitadores) e os de interesse, por hierarquia (forca dos comportamentos) ou por cronologia (seqüência dos comportamentos).

5. Organize suas observações em três colunas: condições antecedentes, comportamento, condições conseqüentes (use como referência para a disposição das condições, cada comportamento identificado).

6. Verifique se não existem lacunas em qualquer das três colunas.

7. Analise o que precisaria ser feito para facilitar (ou para impedir ou dificultar, conforme o caso) a ocorrência do comportamento?

a) Como você alteraria as condições antecedentes?

b) Como você alteraria as condições conseqüentes? As suas alterações implicam em operações de reforçamento ou punição do comportamento de interesse? Implicam em reforçamento de comportamentos incompatíveis?

\section{REFERÊNCIAS BIBLIOGRÁFICAS GERAIS}

HINELINE,P. N. (1980) The language of behavior analysis: Its community, its functions, and its limitations. Behaviorism, vol.ㅁ, pp. 67-86.

JOHNSTON,J. M.\& Pennypackewr, H. S. (1980) Strategies and tactics of human behavioral research. Hillsdale, NJ: Lawrence Erlbaum.

MALOTT, R. W., Whaley, D. L. \& Malott, M. E. (1997) Elementary principies of behavior. Upper Saddle River, NJ: Prentice Hall. 3a edição.

MICHAEL, J. L. (1993) Concepts and Principles of BehaviorAnalysis. Kalamazoo, MI: Society for the Advancement of Behavior Analysis.

SIIDMAN, M. (1960) Tactics of scientific research. New York: Basic Books. Reimpresso em 1988, Boston: Authors Cooperative Inc.
SIDMAN, M. (1994) Equivalence relations and behavior: a Research story. Boston: Authors Cooperative Inc.

SKINNER, B. F. (1931) The concept of the reflex in the description of behavior. Journal of General Psychology, vol. $\underline{5}$, pp. 427-458.

SKINNER, B. F. (1972) A lecture on 'having a poem'. Em Cumulative Record. New York:

Appleton-Century-Crofts, pp. 345-355.

SKINNER, B. F. (1989) The initiating self. Em Recent Issues in the Ana/ysis of Behavior. London: Merril Publishing Co., pp. 27-33.

\section{LEITURAS RECOMENDADAS PARA UMA ANÁLISE FUNCIONAL APLICADA}

Cooper, L. J., Wacker, D. P., Sasso, G. M., Reimers, T. M. \& Donn, L. K. (1990) Using parents as therapists to evaluate appropriate behavior of their children: application to a tertiary diagnostic clinic. Journal of Applied Behavior Analysis, vol. 23, pp. 285-296.

Cooper, L. J., Wacker, D. P., Thursby, D., Plagmann, L. A., Harding, J., Millard, T. \& Derby, M. (1992) Analysis of the effects of tasks preferences, task demands, and adult attention on child behavior in outpatient and classroom settings. Journal of Applied Behavior Analysis, vol. 25, pp. 823-840.

Derby, K. M., Wacker, D. P., Sasso, G., Steege, M., Northup, J., Cigrand, K. \& Asmus, J. (1992) Brief functional assessment techniques to evaluate aberrant behavior in an outpatient setting: asummary of 79 cases. Journal of Applied Behavior Analysis, vol. 25, pp. 713-721.

DuPaul, G. \& Eckert, T. L. (1996) The effects of school-based interventions for Attention Deficit Hyperactivity Disorder: a meta-analysis. School Psychology Review, vo1.26, pp 5-27.

Northup, J., Wacker, D., Sasso, G., Steege, M., Cigrand, K., Cook, J. \& DeRaad, A. (1991) A brief functional analysis of aggressive and alternative behavior in na outclinic setting. 
Journal of Applied Behavior Analysis, vol.24, pp.509-522.

Sasso, G. M., Reimers, T. M., Cooper, L. J., Wacker, D., Berg, W., Steege, M., Kelly, L. \& Allaire, A. (1992) Use of descriptive and experimental analyses to identify the functional properties of aberrant behavior in school settings. Journal of Applied Behavior Analysis, vol. 25, pp. 809-821.

STAGE, S. A. \& Quiroz, D. R. (1997) A metanalysis of interventions to decrease disruptive classroom behavior in public education settings. School Psychology Review, vol.26, pp. 333-368.

WACKER (1990) Use of functional analysis and acceptability measures to asses and treat severe behavior problems: An outpatient clinic model. Em A.C. Repp \& N.N. Singh (Orgs.) Perspectives on the use of nonaversive and aversive interventions for persons with DD. Sycamore, IL: Sycamore Publishing CO., pp. 349-359. 